\title{
SHORT Deletion of glutamate dehydrogenase 1 (Glud1) in COMMUNICATION the central nervous system affects glutamate handling without altering synaptic transmission
}

\author{
Francesca Frigerio, ${ }^{*}, 1$ Melis Karaca, ${ }^{*}, 1$ Mathias De Roo, $\dagger$ Vladimír \\ Mlynárik,ł Dorte M. Skytt, $\S$ Stefania Carobbio,* Kamilla Pajęcka, $\S$ \\ Helle S. Waagepetersen, $\S$ Rolf Gruetter, $\$$ Dominique Muller $†$ and \\ Pierre Maechler* \\ *Department of Cell Physiology and Metabolism, University of Geneva Medical Centre, Geneva, \\ Switzerland \\ $\dagger$ Department of Neuroscience, University of Geneva Medical Centre, Geneva, Switzerland \\ $\ddagger$ Laboratory for Functional and Metabolic Imaging, Ecole Polytechnique Fédérale de Lausanne, \\ Lausanne, Switzerland \\ $\S$ Department of Drug Design and Pharmacology, Faculty of Health and Medical Sciences, University \\ of Copenhagen, Denmark
}

\begin{abstract}
Glutamate dehydrogenase $(\mathrm{GDH})$, encoded by GLUD1, participates in the breakdown and synthesis of glutamate, the main excitatory neurotransmitter. In the CNS, besides its primary signaling function, glutamate is also at the crossroad of metabolic and neurotransmitter pathways. Importance of brain GDH was questioned here by generation of CNSspecific GDH-null mice (CnsGlud $1^{-1-}$ ); which were viable, fertile and without apparent behavioral problems. GDH immunoreactivity as well as enzymatic activity were absent in CnsGlud1 ${ }^{-1-}$ brains. Immunohistochemical analyses on brain sections revealed that the pyramidal cells of control animals were positive for $\mathrm{GDH}$, whereas the labeling was absent in hippocampal sections of Cns-Glud1 $1^{-1-}$ mice. Electrophysio-
\end{abstract}

logical recordings showed that deletion of GDH within the CNS did not alter synaptic transmission in standard conditions. Cns-Glud1 $1^{-1-}$ mice exhibited deficient oxidative catabolism of glutamate in astrocytes, showing that GDH is required for Krebs cycle pathway. As revealed by NMR studies, brain glutamate levels remained unchanged, whereas glutamine levels were increased. This pattern was favored by upregulation of astrocyte-type glutamate and glutamine transporters and of glutamine synthetase. Present data show that the lack of GDH in the CNS modifies the metabolic handling of glutamate without altering synaptic transmission.

Keywords: astrocytes, central nervous system, Glud1, glutamate, glutamate dehydrogenase.

J. Neurochem. (2012) 123, 342-348.
Glutamate is the major excitatory neurotransmitter in mammalian CNS and is involved in mechanisms of synaptic plasticity, memory, and neuronal or glial cell death. Upon glutamatergic transmission, intersynaptic glutamate clearance is achieved mostly by astrocytes (Bak et al. 2006), preventing toxic accumulation in the extracellular space. In the astrocytes, glutamate is either amidated to glutamine via glutamine synthetase and then recycled back to neurons as part of glutamate-glutamine cycle. Alternatively, glutamate is
Received April 14, 2012; revised manuscript received July 30, 2012; accepted August 15, 2012.

Address correspondence and reprint requests to Pierre Maechler, Department of Cell Physiology and Metabolism, University of Geneva Medical Centre, 1 rue Michel-Servet, 1211 Geneva 4, Switzerland. Email: pierre.maechler@unige.ch

${ }^{1}$ F.F. and M.K. contributed equally to this work.

Abbreviations used: DMEM, Dulbecco's modified Eagle's medium; EAAT, excitatory amino acid transporter; fEPSPs, extracellular field excitatory post-synaptic potentials; GDH, glutamate dehydrogenase; LTP, long-term potentiation; TCA, tricarboxylic acid. 
deaminated to yield $\alpha$-ketoglutarate, followed by further oxidation in the tricarboxylic acid (TCA) cycle to generate ATP (Yu et al. 1982; McKenna et al. 1996). Such an essential process is commonly attributed to both glutamate dehydrogenase (GDH) and transaminases, although their relative importance has not been established yet. GDH, in rodents encoded solely by GLUD1 (Michaelidis et al. 1993), is a mitochondrial enzyme catalyzing either the anabolic reductive amination of $\alpha$-ketoglutarate producing glutamate or, conversely, the catabolic oxidative deamination of glutamate (Karaca et al. 2011). In CNS, GDH is predominantly expressed in astrocytes and to lower levels in neurons (Rothe et al. 1994; Mastorodemos et al. 2005). To date, the putative importance of GDH as a player in balanced glutamateglutamine cycle activity and as a supplier of precursors for glutamatergic neurotransmission remains to be established, essentially because we lack loss-of-function models.

Abnormal glutamatergic transmission has been associated with major neurodegenerative diseases (Kim et al. 2011). Accordingly, attenuation of glutamate toxicity is foreseen as one possible therapeutic strategy. Recent characterization of transgenic mice over-expressing Glud1 in CNS neurons showed enhanced glutamate release and neuronal damages (Bao et al. 2009). In this context, GDH represents a promising target for the study of glutamate-associated neurodegenerative diseases.

This study reports the first mouse model with brain-specific ablation of GDH, named Cns-Glud1 ${ }^{-/-}$.We questioned the putative importance of GDH in synaptic transmission and in the maintenance of glutamate-glutamine balance. Our data show that the lack of Gludl in brain inhibited GDH activity regardless of enzymatic direction, modified glutamate handling, decreased glutamate catabolism to $\mathrm{CO}_{2}$, and increased glutamine levels; without affecting synaptic transmission.

\section{Material and methods}

Brain-specific GDH knockout mouse Cns-Glud1 ${ }^{-1-}$

Glud1 floxed (Gludl lox/lox) mice (Carobbio et al. 2009) were crossed with mice expressing Cre recombinase under the control of nestin cis-regulatory sequence (Tronche et al. 1999). Heterozygous Cns-Glud1 ${ }^{-/+}$were crossed with Glud1 ${ }^{\text {lox/lox }}$ mice to obtain homozygous Cns-Glud1 ${ }^{-/-}$. Animals were maintained on a mixed (C57BL/6J x 129/Sv) genetic background to avoid inbred strainspecific phenotypes. As control mice, we used Gludl ${ }^{\text {lox/lox }}$ littermates of Cns-Glud1 ${ }^{-1-}$ to optimize standardization of the genetic background between the two groups. Both male and female animals were studied for some representative parameters. As no gender differences were noticed, detailed analyses presented here are only from males. Mice were maintained in our animal facility according to procedures approved by the animal care and experimentation authorities of the Canton of Geneva.

\section{GDH expression and activity assessment}

Gludl deletion in knockout brains was assessed at the protein level by immunoblotting on tissues collected from killed mice in radioimmunoprecipitation assay buffer. Protein extracts were separated in bis-acrylamide $10 \%$ gel and probed with anti-GDH (Rockland, Gilbertsville, PA, USA) and anti-actin (Millipore, Billerica, MA, USA) primary antibodies as described (Carobbio et al. 2004). Bands were revealed by horseradish peroxidase detection system. GDH enzymatic activity was measured in brains collected in ice-cold saline before homogenization in $50 \mathrm{mM}$ Tris $/ \mathrm{HCl} \mathrm{pH} 7.4,0.5 \mathrm{mM}$ EDTA, $1 \mathrm{mM} \mathrm{B-mercaptoethanol} \mathrm{in} \mathrm{glass} \mathrm{potter.} \mathrm{After} 40 \mathrm{~min}$ centrifugation at $8000 \mathrm{~g}$, supernatants were assayed for enzymatic activity (Carobbio et al. 2004). For immunohistochemistry, hippocampi were fixed overnight in $4 \%$ paraformaldehyde, dehydrated and embedded in paraffin. Sections $(5 \mu \mathrm{m})$ treated with citrate buffer, $\mathrm{pH}$ 6, were incubated overnight with rabbit anti-GDH (Rockland) antibody at $4^{\circ} \mathrm{C}$. They were then incubated with Alexa 488-conjugated goat anti-rabbit (Invitrogen, Carlsbad, CA, USA) antibody and analyzed by confocal microscopy using a 60x objective (Olympus FV300 confocal LSM, Hamburg, Germany).

\section{Electrophysiology}

Hippocampal slices $(400 \mu \mathrm{m})$ were prepared from 4.5 - to 5.5-monthold Cns-Glud1 $1^{-/-}$and Glud1 ${ }^{\text {lox/lox }}$ control mice as described previously (Quintana et al. 2006). Slices were maintained in an interface chamber at $32^{\circ} \mathrm{C}\left(95 \% \mathrm{O}_{2}, 5 \% \mathrm{CO}_{2}\right)$ and perfused with buffer containing (in mM): $124 \mathrm{NaCl}, 1.6 \mathrm{KCl}, 2.5 \mathrm{CaCl}_{2}, 1.5 \mathrm{MgCl}_{2}, 24$ $\mathrm{NaHCO}_{3}, 1.2 \mathrm{KH}_{2} \mathrm{PO}_{4}, 10$ glucose, and 2 ascorbic acid, $\mathrm{pH}$ 7.4. Extracellular field excitatory post-synaptic potentials (fEPSPs) were evoked by a bipolar stimulating electrode made with two twisted strands of nichrome wires $(50 \mu \mathrm{m}$ of diameter), triggered with a Master-8 (AMPI, Jerusalem, Israel) and placed on a group of Schaffer collaterals. fEPSPs were evoked by stimulation of a group of Schaffer collaterals and recorded in the stratum radiatum of CA1 using an Axoclamp 2A amplifier (Molecular Devices, Sunnyvale, CA, USA). Paired-pulse facilitation was elicited by repeated pulses at $50 \mathrm{~ms}$ interval. Input/output relationship was determined by monitoring responses to increased stimulation intensities. Long-term potentiation (LTP) was induced by theta burst stimulation (five trains at $5 \mathrm{~Hz}$ composed each of four pulses at $100 \mathrm{~Hz}$, repeated twice at $10 \mathrm{~s}$ intervals) and the level of potentiation assessed by measuring the increase in averaged amplitude and slope of 10 responses of the baseline, with 10 responses evoked 60 min after stimulation.

\section{Cultures of astrocytes}

Astrocytes were cultured from dissociated cerebella of 7-day-old mice as detailed previously (Hertz et al. 1989). To obtain single-cell suspension, cerebella were dissected and mechanically dissociated by passing the tissue through $80 \mu \mathrm{m}$ nylon sieves into Dulbecco's modified Eagle's medium (DMEM; Sigma-Aldrich, St. Louis, MO, USA) supplemented with $6 \mathrm{mM}$ glucose, $2.5 \mathrm{mM}$ glutamine, and $20 \%$ fetal bovine serum. The cell suspension was seeded in $25 \mathrm{~cm}^{2}$ flasks, corresponding to 0.8 mice/flask. Cells were maintained in culture for 3 weeks prior to experiments and serum was reduced to $15 \%$ the second week. Then, astrocytes were trypsinized and replated in 24- or 6-well plates and maintained for the third week in a medium containing $10 \%$ serum. Stellation of the cells was induced by $0.25 \mathrm{mM}$ dibutyryl-cAMP (Sigma-Aldrich).

$\mathrm{CO}_{2}$ production by astrocytes from glutamate

$\mathrm{CO}_{2}$ production was determined as previously described (Frigerio et al. 2010). Prior to the experiment, the astrocytes were incubated 
for $1 \mathrm{~h}$ in serum-free DMEM without glucose and glutamine. Astrocytes were subsequently incubated in DMEM containing $2.5 \mathrm{mM}$ glucose and $250 \mu \mathrm{M}$ labeled glutamate $(0.1 \mu \mathrm{Ci}$ of $\mathrm{L}-$ [U- ${ }^{14}$ C]glutamate, \#0165A American Radiolabeled Chemicals Inc., St.Louis, MO, USA) for $1 \mathrm{~h}$ at $37^{\circ} \mathrm{C}$. During the incubation, generated $\mathrm{CO}_{2}$ was trapped on $3 \mathrm{MM}$ chromatography paper soaked in $2 \mathrm{M} \mathrm{NaOH}$. Metabolic activity was stopped by placing the plates at $-20^{\circ} \mathrm{C}$. The next day, ${ }^{14} \mathrm{CO}_{2}$ radioactivity was quantified in a liquid scintillation $B$-counter and protein from cell extracts determined for normalization using Bradford assay.

NMR measurements of brain metabolites

Anesthesia was maintained at $1.3 \pm 0.2 \%$ of isoflurane in oxygen, body temperature was kept at $36.5 \pm 0.2^{\circ} \mathrm{C}$. Spectra were acquired on an actively shielded $9.4 \mathrm{~T} / 31 \mathrm{~cm}$ INOVA animal scanner (Varian/Magnex Scientific, Palo Alto, CA, USA) using the SPECIAL spectroscopy sequence (Mlynarik et al. 2006). This technique enables to measure short-echo-time spectra with enhanced signal intensity from the excited volume. A 14-mm-diameter twoloop quadrature coil was used both for RF excitation and signal reception. Field homogeneity was adjusted using the FASTMAP protocol (Gruetter and Tkac 2000). A VOI having the nominal size of 22-30 $\mathrm{LL}$ (typically $3 \mathrm{~mm} \times 2.5 \mathrm{~mm} \times 3 \mathrm{~mm}$ ) was selected in striatum, TR was $4 \mathrm{~s}$ and TE was $2.7 \mathrm{~ms}$. Signal from the outer volume was suppressed by four blocks of slice-selective pulses, water signal was suppressed by the VAPOR sequence (Tkac et al. 1999). The 160 scans collected in 10 min provided spectra with excellent SNR. Absolute concentrations of metabolites were obtained by LCModel using unsuppressed water signal as a reference resulting in a neurochemical profile of more than 18 metabolites. The Cramer-Rao lower bounds for concentrations of the most abundant metabolites were $1-2 \%$.

Expression analysis by quantitative RT-PCR and immunoblotting Total RNA was extracted from brains collected from control and CnsGlud1 $1^{-1-}$ mice by peqGOLD Trifast (Peqlab, Erlangen, Germany). Target genes were amplified by Real-Time PCR (StepOnePlus, Applied Biosystems, Foster City, CA, USA), normalized to cyclophillin levels. Following primers were used (forward and reverse primers): glutamine synthetase, 5'-CAGGCTGCCATACCAACTTCA-3', 5'- TG GCCTCCTCAATGCACTTC-3'; excitatory amino acid transporter 1 (EAAT1), 5'-TAACGAAGCCATCATGCGATT-3', 5'-CCCTGCGAT CAAGAAGAGGAT-3'; EAAT2, 5'-AGGTCAGATTGTGACTGTA AGCCTTA-3', 5'-CCGGCGCTGGGAATACT-3'; SNAT3, 5'-GCTGC CCATATATACAGAGCTCAAG-3', 5'-GACAGCAATGGACAGG TTGGA-3'; SNAT7, 5'-GTGTGCTCACCTCCTATCCAATCT-3', 5'-CACGGGCATCCCCTTGTAG-3'; glutaminase, 5'-TCAAAGG GTGAAGTCGGTGAT-3', 5'- AATCCC GCTGCTCCATGTC-3'; vesicular glutamate transporter 1 (vGLUT1), 5'-GGAGGCCAGAT CGCTGACT-3', 5'-AGCTTTCGCACGTTGGTAGTG-3'; cyclophillin, 5'-ATGGCAAATGCTGGACCAA-3', 5'-GCCATCCAGC CATTCAGTCT-3'.

At the protein level, immunoblotting analyses were performed on tissues collected in ristocetin-induced platelet agglutination buffer as described above. Following bis-acrylamide gel electrophoresis and transfer, proteins were detected by using primary antibodies against glutamine synthetase (Millipore), EAAT2/SLC1A2 (Cell signaling, Beverly, MA, USA), SNAT3/SLC38A3 (Proteintech, Chicago, IL,
USA), and ezerin (Santa Cruz Biotechnology, Santa Cruz, CA, USA) serving as loading control.

\section{Statistical analysis}

Unless otherwise stated, such as for electrophysiology, statistical significance was determined by Student's $t$-test. A $p$ value $<0.05$ was used to indicate a significant difference.

\section{Results}

Assessment of brain-specific GDH-knockout mouse, Cns-Glud $1^{-/-}$

Cns-Glud1 $1^{-/-}$mice were generated by crossing Glud1 ${ }^{\text {lox/lox }}$ with Nestin-Cre mice. Deletion was successful and specific for brain, as GDH expression was fully preserved in other tissues known to express GDH (Fig. 1a). We observed abrogation of GDH enzymatic activity in brain homogenates of Cns-Glud1 $1^{-1-}$, both in oxidative deamination and in reductive amination directions (Fig. $1 \mathrm{~b}$ and c). The hippocampal CA1 region, rich in glutamatergic synapses, was analyzed by immunohistochemistry. Dense GDH labeling was observed in controls, while the signal was absent in hippocampal sections of Cns-Glud1 $1^{-1-}$ mice (Fig. 1d). Homozygous mice were viable and fertile and did not exhibit apparent behavioral problems when handling animals for regular cares on a daily basis over a period of more than 4 years.

\section{Ablation of GDH in brain does not affect synaptic} transmission

The fEPSPs showed identical electrophysiological properties for $\mathrm{Cns}-\mathrm{Glud1}^{-/-}$and control mice, regarding synaptic transmission and synaptic plasticity. As shown in Fig. 2a-d, evoked fEPSPs were not different in terms of time (decay: $6.49 \pm 0.59$ vs. $7.00 \pm 0.78 \mathrm{~ms}, p=0.36$, unpaired $t$-test), paired-pulse facilitation $(37.2 \pm 3.1$ vs. $36.0 \pm 7.6 \%$, $p=0.34$, unpaired $t$-test) as well as for the input-output relationship of their amplitude and slope ( $p$ values of 0.67 and 0.36, respectively; two-way ANOva with Bonferroni posttest). In addition, Cns-Glud1 ${ }^{-1-}$ mice showed normal LTP (Fig. 2e) with similar rates of success compared with control mice (Fisher's exact test, Fig. 2f) and similar levels of fEPSP slope potentiation $(51 \pm 31$ vs. $46 \pm 18 \%$, respectively, $p=1$, Mann-Whithney $U$-test, Fig. $2 \mathrm{~g}$ ). Overall, these electrophysiological recordings showed that deletion of GDH within the CNS did not alter synaptic transmission in standard conditions. However, it remains to be determined if chronic glutamate exposure would result in similarly unaltered glutamatergic activity.

Glutamate handling is affected in Cns-Glud $1^{-/}$brain Glutamate may serve as energy substrate via GDH-dependent deamination to yield $\alpha$-ketoglutarate followed by further oxidation in the TCA cycle (Fig. 3a). Oxidative catabolism 
(a)

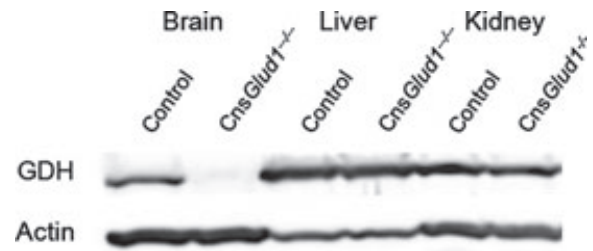

(b)

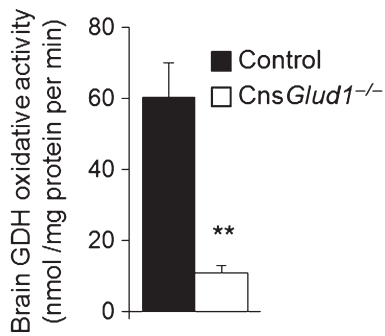

(d)

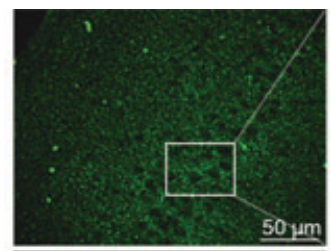

(c)
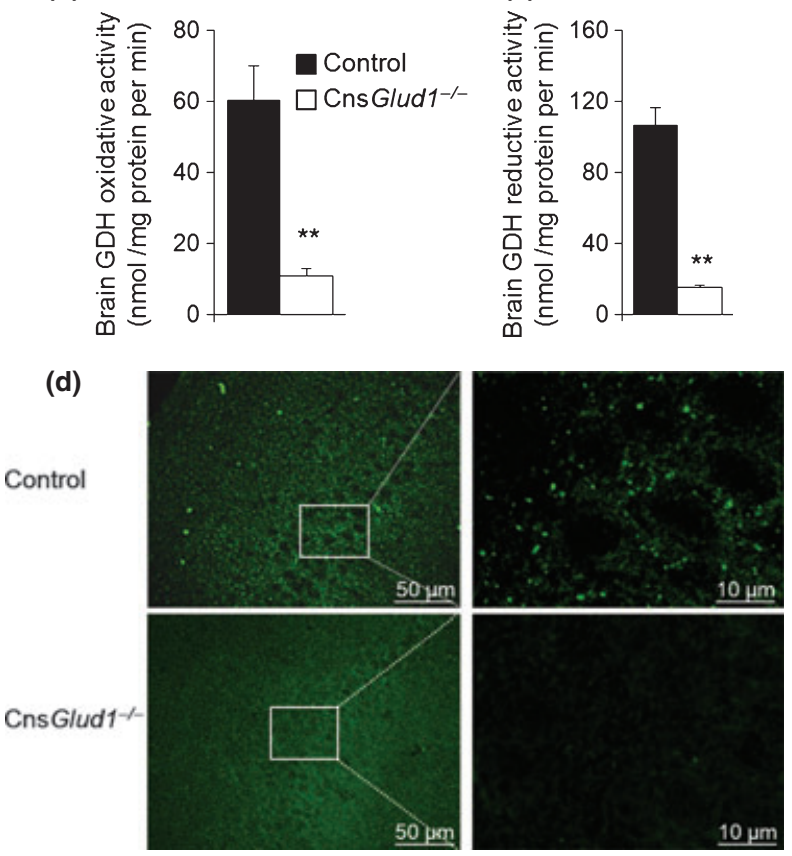

Fig. 1 Assessment of brain-specific glutamate dehydrogenase (GDH)knockout mouse, Cns-Glud1 ${ }^{-/-}$. (a) Brain-specific Glud1 deletion was controlled by immunoblotting for GDH expression and compared to non-excitable tissues collected from control (Glud1 $1^{\text {lox/lox}}$ ) and CNSspecific Glud1-knockout Cns-Glud1 ${ }^{-1-}$ mice. Actin served as loading control. Immunoblots are representative of three independent preparations. (b, c) Enzymatic activity of GDH measured in brain extracts of control and Cns-Glud1 ${ }^{-1-}$ mice measured in both oxidative (b) and reductive (c) directions, using glutamate and $\alpha$-ketoglutarate as substrates, respectively. Values are means $\pm \mathrm{SE}, n=5, * * p<0.01$ versus control. (d) Representative immunohistochemical labeling for $\mathrm{GDH}$ on $\mathrm{CA} 1$ field of the hippocampus. Right panels are 5x enlargements of left panels.

of glutamate was reduced by about $30 \%$ in $\mathrm{Cns}-\mathrm{Glud1}^{-1-}$ astrocytes compared to controls, as measured by ${ }^{14} \mathrm{CO}_{2}$ production from $\left[\mathrm{U}_{-}{ }^{14} \mathrm{C}\right]$ glutamate (Fig. 3b). Thus, the lack of glutamate oxidation via GDH was not compensated by transaminase pathways. Of note, intrabrain levels of aminotransferase substrates alanine and aspartate were not changed in Cns-Glud1 $1^{-1-}$ animals, as measured by NMR (Fig. 3c).

Interestingly, intrabrain glutamate levels were similar between control and Cns-Glud1 $1^{-1-}$ mice (Fig. 3c). An alternative metabolic fate for glutamate in astrocytes is its amidation to glutamine, catalyzed by glutamine synthetase. Then, part of newly synthesized glutamine leaves the astro- cytes, mainly through specific glutamine transporter SNAT3 (Fig. 3a). We observed higher intrabrain concentrations of glutamine in Cns-Glud1 ${ }^{-1-}$ mice (Fig. 3c) accompanied by increased expression of both glutamine synthetase and astrocyte-specific glutamate transporters EAAT1 and EAAT2, as well as the glutamine transporter SNAT3 (Fig. 3d and e). These results indicate that in cultured astrocytes originating from Cns-Glud1 ${ }^{-1-}$ mice and exhibiting reduced $\mathrm{CO}_{2}$ production from glutamate, glutamate metabolism may be directed toward glutamine synthesis and export.

Glutamine synthesized by astrocytes is taken up by neurons, notably through recently identified neuronal glutamine transporter SNAT7 (Hagglund et al. 2011). Glutamine is then converted back to glutamate in pre-synaptic terminals by the action of glutaminase before translocation into secretory vesicles via vGLUT1 (Fig. 3a). In Cns-Glud1 $1^{-1-}$ mice, the expression of glutaminase and vGLUT1 remained unchanged (Fig. 3d). Finally, intrabrain GABA concentrations were comparable between control and Cns-Glud1 ${ }^{-1-}$ mice, arguing against accumulation of this decarboxylation product of glutamate (Fig. 3c).

Altogether, these data show that the lack of GDH in brain affects glutamate handling mainly in astrocytes, decreasing glutamate catabolism and increasing intrabrain glutamine levels.

\section{Discussion}

Glutamate is the major excitatory neurotransmitter in the brain, with about $40 \%$ of all synapses being glutamatergic (Fairman and Amara 1999). Glutamate may also exert neurotoxic actions leading to neuronal death. Accumulation of extracellular glutamate and subsequent over-stimulation of glutamatergic receptors is harmful. Thus, tight glutamate handling in the CNS is crucial for maintenance of its integrity. Abnormalities in this process are associated with several neurodegenerative diseases; such as Parkinson's disease, epilepsy, schizophrenia, and Alzheimer's disease (Kim et al. 2011). Animal models lacking glial glutamate transporter Eaat2 (Slcla2, Glt-1) exhibit accumulation of extracellular glutamate and extensive brain damages (Rothstein et al. 1996; Tanaka et al. 1997). Regarding GDH, higher expression has been reported in Alzheimer's brains (Burbaeva et al. 2005; Owen et al. 2009) and lower activity in temporal cortex of epileptic patients (Malthankar-Phatak et al. 2006).

Initial insights into Cns-Glud1 ${ }^{-1-}$ mice show that (i) both oxidative deamination and reductive amination activities of GDH were inhibited, (ii) GDH abrogation did not affect synaptic transmission, (iii) intrabrain levels of glutamate remained similar to controls. Cultured astrocytes incubated with labeled glutamate revealed that the lack of GDH impaired glutamate catabolism in the TCA cycle, as demonstrated by lower $\mathrm{CO}_{2}$ production. Interestingly, this was not compensated by transamination pathways. It should be noted that results obtained from measurements of glutamate- 
(a)

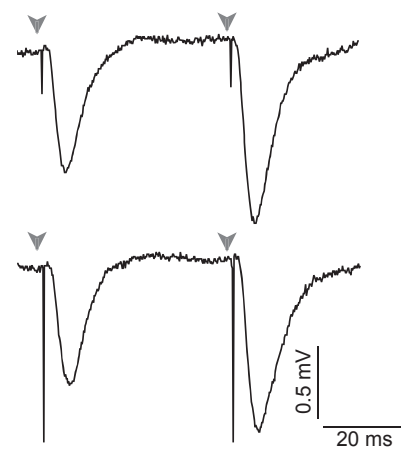

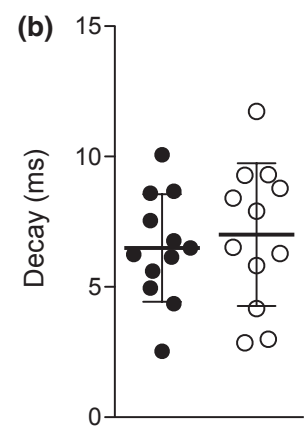

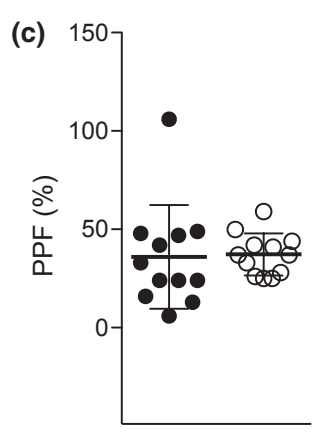

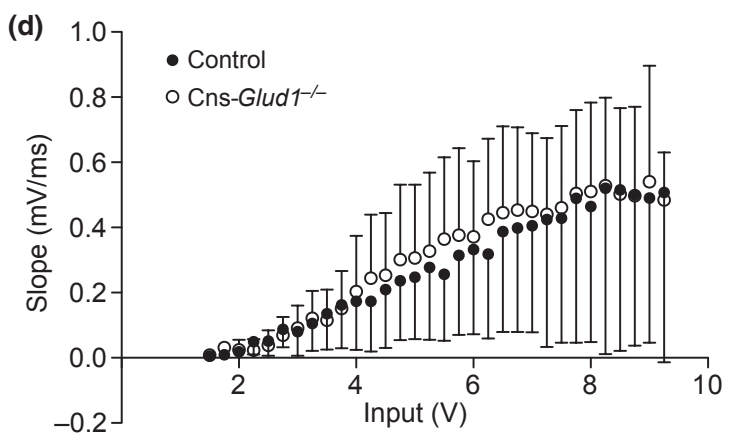
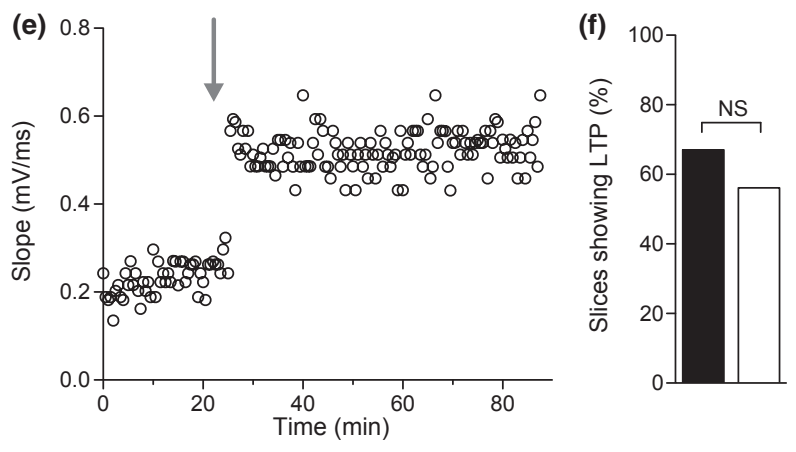

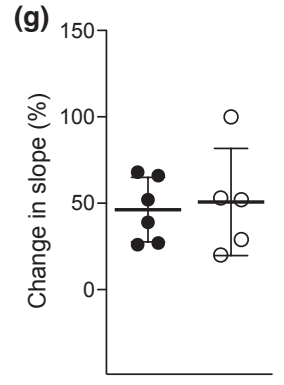

Fig. 2 Ablation of Glutamate dehydrogenase (GDH) in brain does not affect synaptic transmission. (a) Illustration of two evoked field excitatory post-synaptic potentials (fEPSPs) separated by $50 \mathrm{~ms}$ in control (Ctrl) and Cns-Glud1 ${ }^{-1-}$ (KO) hippocampal slices, revealing similar levels of paired-pulse facilitation. Arrowheads indicate stimulation of Schaffer collaterals. (b) Decay time of evoked fEPSPs recorded in control versus Cns-Glud1 ${ }^{-1-}$ mice. (c) Magnitude of paired-pulse facilitation (PPF, in \%) of evoked fEPSPs recorded in control versus Cns-Glud1 ${ }^{-1-}$ mice. (d) Input-ouput curve of fEPSP slope recorded in control versus Cns-Glud1 ${ }^{-1-}$ mice. (e) Representative Long-Term

derived $\mathrm{CO}_{2}$ did not take into account a potentially elevated glutamate uptake by Cns-Glud1 ${ }^{-1-}$ astrocytes, indicated by the augmented EAAT expression in the whole brain. Enhanced glutamate uptake would lead to higher specific radioactivity of intracellular glutamate in Cns-Glud1 ${ }^{-1-}$ astrocytes, and thus, the reduced $\mathrm{CO}_{2}$ production observed in these astrocytes might be underestimated. Nevertheless, the reduced capacity of glutamate catabolism did not result in
Potentiation (LTP) experiment performed on a hippocampal slice of a Cns-Glud $1^{-1-}$ animal. Dots represent values of fEPSP slope recorded every $30 \mathrm{~s}$. LTP was induced by theta burst stimulation (arrow). (f) Proportion of slices that showed a least a $20 \%$ increase in EPSP slope 60 min after stimulation in control and Cns-Glud1 $1^{-1-}$ animals. (g) Changes in EPSP slope (in \%) measured 60 min after stimulation in control and Cns-Glud1 ${ }^{-1-}$ mice. (b-d): values are means \pm SD, $n=12$ slices out of five animals in each group. (f-g): $n=9$ slices out of four animals in each group.

accumulation of glutamate in Cns-Gludl $1^{-1-}$ brains. Instead, glutamate might be redirected to glutamine synthesis and export out of the astrocytes, as indicated by the observed up-regulation of astrocyte-type glutamine transporter SNAT3 and glutamine synthetase in brains of Cns-Glud1 ${ }^{-1-}$ mice.

Before this first mouse model of brain-specific loss-offunction of GDH, Bao and colleagues reported the opposite maneuver by generating transgenic mice over-expressing 
(a)

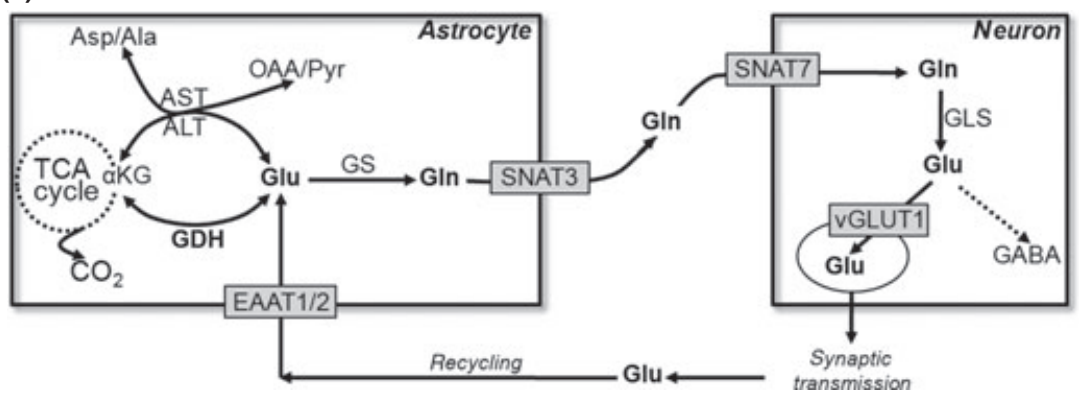

(b) Control 口 CnsGlud1-1-

(c)
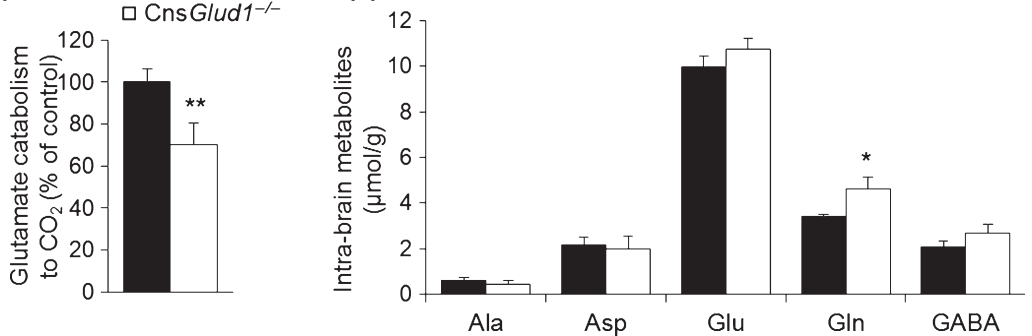

(d)

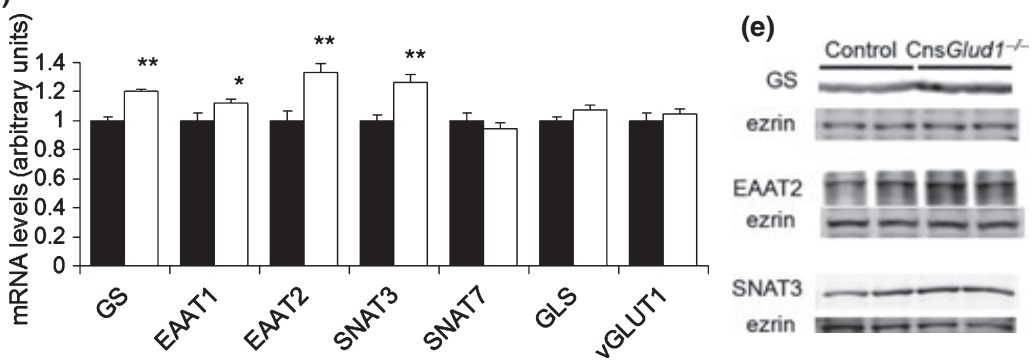

Fig. 3 Glutamate handling is affected in Cns-Glud1 $1^{-/}$brain. (a) Simplified representation of glutamate fates in astrocytes and neurons ( $\alpha$-ketoglutarate, $\alpha \mathrm{KG}$; alanine, Ala; alanine aminotransferase, ALT; aspartate, Asp; aspartate aminotransferase, AST; excitatory amino acid transporters 1/2, EAAT 1/2; glutamate dehydrogenase, GDH; glutamine, Gln; glutaminase, GLS; glutamate, Glu; glutamine synthetase, GS; oxaloacetate, OA; pyruvate, Pyr; sodium-coupled amino acid transporters $3 / 7$, SNAT $3 / 7$; vesicular glutamate transporter 1 , vGLUT1). (b) Glutamate catabolism was determined in astrocytes from control and Cns-Glud1 $1^{-1-}$ mice by measuring ${ }^{14} \mathrm{CO}_{2}$ production from L-[U- ${ }^{14}$ C]glutamate $(250 \mu \mathrm{M}) ; n=7, * * p<0.01$ versus control. (c)

GDH in neurons (Bao et al. 2009). In this gain-of-function model, GDH levels and activity are increased by about $30 \%$ and $40 \%$, respectively. These mice exhibit increased depolarization-evoked glutamate release, increased frequency and amplitude of hippocampal spontaneously occurring synaptic events (mEPSC), diminished LTP of synaptic activity, and age-associated neuronal losses (Bao et al. 2009).

In the CNS, glutamate de novo synthesis is mainly contributed by glucose as the source of precursors for the TCA cycle intermediate, $\boldsymbol{\alpha}$-ketoglutarate, via pyruvate carboxylation in astrocytes. However, the amination forming glutamate has been suggested to occur via either GDH or
NMR analysis of brain metabolites in control and Cns-Glud1 $1^{-1-}$ mice; $n=11, * p<0.05$ versus control. (d) Gene expression of enzymes and transporters implicated in CNS glutamate pathways in brains of control and Cns-Glud $1^{-1-}$ mice. Values are normalized to cyclophillin; $n=4-6$, $* p<0.05$ and $* * p<0.01$ versus control. (b-d): Values are means \pm SE. (e) Representative immunoblotting showing protein levels of genes exhibiting the major changes at mRNA levels (d) in control and Cns-Glud1 ${ }^{-1-}$ brains (changes on average versus control: GS, $+28 \%, p<0.01 ;$ EAAT2, $+38 \%, p<0.05 ;$ SNAT3, $+34 \%$ $p<0.01 ; n=5-6$ mice). Ezerin served as loading control and each lane corresponds to brain extract from one mouse.

transamination pathways, i.e., branched-chain aminotransferases. In the over-expression model, increased GDH levels specifically in neurons led to an increased production of glutamate (Bao et al. 2009). In the Cns-Glud1 ${ }^{-1-}$ model lacking GDH, such a pathway was abrogated and we additionally observed reduced glutamate breakdown. There was neither over-excitability nor hypo-excitability, indicating that glutamate homeostasis was maintained, pending increased formation of glutamine. Present data on CnsGlud1 $1^{-1-}$ model highlight the fine tuning and compartmentalization of glutamate-glutamine handling and open new avenues for further studies investigating the contribution of GDH in neurodegenerative diseases. 


\section{Acknowledgements}

This study was supported by the State of Geneva, the Centre d'imagerie biomedicale (CIBM of UNIL, UNIGE, EPFL, HUG, and CHUV), the Swiss National Science Foundation (310030B-135704 to P.M. and 31003A-127552 to D.M.), a fellowship from the AXA Research Fund (to M.K.), the Danish Medical Research Council (09-063399 to D.M.S. and 09-066319 to K.P.), the Lundbeck Foundation (to H.S.W), the Leenaards and Jeantet Foundations (to V.M. and R.G.). We thank Pedro L. Herrera for help with nestinCre mice. The authors declare no conflicts of interest.

\section{References}

Bak L. K., Schousboe A. and Waagepetersen H. S. (2006) The glutamate/GABA-glutamine cycle: aspects of transport, neurotransmitter homeostasis and ammonia transfer. J. Neurochem. 98, 641-653.

Bao X., Pal R., Hascup K. N. et al. (2009) Transgenic expression of Glud1 (glutamate dehydrogenase 1) in neurons: in vivo model of enhanced glutamate release, altered synaptic plasticity, and selective neuronal vulnerability. J. Neurosci. 29, 13929-13944.

Burbaeva G., Boksha I. S., Tereshkina E. B., Savushkina O. K., Starodubtseva L. I. and Turishcheva M. S. (2005) Glutamate metabolizing enzymes in prefrontal cortex of Alzheimer's disease patients. Neurochem. Res. 30, 1443-1451.

Carobbio S., Ishihara H., Fernandez-Pascual S., Bartley C., Martin-DelRio R. and Maechler P. (2004) Insulin secretion profiles are modified by overexpression of glutamate dehydrogenase in pancreatic islets. Diabetologia 47, 266-276.

Carobbio S., Frigerio F., Rubi B. et al. (2009) Deletion of Glutamate Dehydrogenase in Beta-Cells Abolishes Part of the Insulin Secretory Response Not Required for Glucose Homeostasis. J. Biol. Chem. 284, 921-929.

Fairman W. A. and Amara S. G. (1999) Functional diversity of excitatory amino acid transporters: ion channel and transport modes. Am. J. Physiol. 277, F481-F486.

Frigerio F., Brun T., Bartley C., Usardi A., Bosco D., Ravnskjaer K., Mandrup S. and Maechler P. (2010) Peroxisome proliferatoractivated receptor alpha (PPARalpha) protects against oleateinduced INS-1E beta cell dysfunction by preserving carbohydrate metabolism. Diabetologia 53, 331-340.

Gruetter R. and Tkac I. (2000) Field mapping without reference scan using asymmetric echo-planar techniques. Magn. Reson. Med. 43, 319-323.

Hagglund M. G., Sreedharan S., Nilsson V. C., Shaik J. H., Almkvist I. M., Backlin S., Wrange O. and Fredriksson R. (2011) Identification of SLC38A7 (SNAT7) protein as a glutamine transporter expressed in neurons. J. Biol. Chem. 286, 20500-20511.

Hertz L., Juurlink B. H. J., Hertz E., Fosmark H. and Schousboe A. (1989) Preparation of primary cultures of mouse (rat) astrocytes, in A dissection and tissue culture manual of the nervous system, (Shaher A., De Vellis J., Vernadakis A. and Haber B., eds.), pp. 92-104. New York, Alan R. Liss, Inc.
Karaca M., Frigerio F. and Maechler P. (2011) From pancreatic islets to central nervous system, the importance of glutamate dehydrogenase for the control of energy homeostasis. Neurochem. Int. 59, 510-517.

Kim K., Lee S. G., Kegelman T. P. et al. (2011) Role of excitatory amino acid transporter-2 (EAAT2) and glutamate in neurodegeneration: opportunities for developing novel therapeutics. J. Cell. Physiol. 226, 2484-2493.

Malthankar-Phatak G. H., de Lanerolle N., Eid T., Spencer D. D., Behar K. L., Spencer S. S., Kim J. H. and Lai J. C. (2006) Differential glutamate dehydrogenase (GDH) activity profile in patients with temporal lobe epilepsy. Epilepsia 47, 1292-1299.

Mastorodemos V., Zaganas I., Spanaki C., Bessa M. and Plaitakis A. (2005) Molecular basis of human glutamate dehydrogenase regulation under changing energy demands. J. Neurosci. Res. 79, $65-73$.

McKenna M. C., Sonnewald U., Huang X., Stevenson J. and Zielke H. R. (1996) Exogenous glutamate concentration regulates the metabolic fate of glutamate in astrocytes. J. Neurochem. 66, 386-393.

Michaelidis T. M., Tzimagiorgis G., Moschonas N. K. and Papamatheakis J. (1993) The human glutamate dehydrogenase gene family: gene organization and structural characterization. Genomics 16, 150-160.

Mlynarik V., Gambarota G., Frenkel H. and Gruetter R. (2006) Localized short-echo-time proton MR spectroscopy with full signal-intensity acquisition. Magn. Reson. Med. 56, 965-970.

Owen J. B., Di Domenico F., Sultana R., Perluigi M., Cini C., Pierce W. M. and Butterfield D. A. (2009) Proteomics-determined differences in the concanavalin-A-fractionated proteome of hippocampus and inferior parietal lobule in subjects with Alzheimer's disease and mild cognitive impairment: implications for progression of AD. J. Proteome Res. 8, 471-482.

Quintana P., Alberi S., Hakkoum D. and Muller D. (2006) Glutamate receptor changes associated with transient anoxia/hypoglycaemia in hippocampal slice cultures. Eur. J. Neurosci. 23, 975-983.

Rothe F., Brosz M. and Storm-Mathisen J. (1994) Quantitative ultrastructural localization of glutamate dehydrogenase in the rat cerebellar cortex. Neuroscience 62, 1133-1146.

Rothstein J. D., Dykes-Hoberg M., Pardo C. A. et al. (1996) Knockout of glutamate transporters reveals a major role for astroglial transport in excitotoxicity and clearance of glutamate. Neuron 16, 675-686.

Tanaka K., Watase K., Manabe T. et al. (1997) Epilepsy and exacerbation of brain injury in mice lacking the glutamate transporter GLT-1. Science 276, 1699-1702.

Tkac I., Starcuk Z., Choi I. Y. and Gruetter R. (1999) In vivo 1H NMR spectroscopy of rat brain at $1 \mathrm{~ms}$ echo time. Magn. Reson. Med. 41, 649-656.

Tronche F., Kellendonk C., Kretz O., Gass P., Anlag K., Orban P. C., Bock R., Klein R. and Schutz G. (1999) Disruption of the glucocorticoid receptor gene in the nervous system results in reduced anxiety. Nat. Genet. 23, 99-103.

Yu A. C., Schousboe A. and Hertz L. (1982) Metabolic fate of 14Clabeled glutamate in astrocytes in primary cultures. J. Neurochem. 39, 954-960. 